\title{
Hawking Radiation-Quasi-Normal Modes Correspondence and Effective States for Nonextremal Reissner-Nordström Black Holes
}

\author{
C. Corda, ${ }^{1,2,3}$ S. H. Hendi, ${ }^{4,5}$ R. Katebi, ${ }^{6}$ and N. O. Schmidt ${ }^{7}$ \\ ${ }^{1}$ Dipartimento di Fisica e Chimica, Università Santa Rita and Institute for Theoretical Physics and \\ Advanced Mathematics Einstein-Galilei (IFM), 59100 Prato, Italy \\ ${ }^{2}$ International Institute for Applicable Mathematics \& Information Sciences (IIAMIS), Hyderabad, India \\ ${ }^{3}$ International Institute for Applicable Mathematics \& Information Sciences (IIAMIS), Udine, Italy \\ ${ }^{4}$ Physics Department and Biruni Observatory, College of Sciences, Shiraz University, Shiraz 71454, Iran \\ ${ }^{5}$ Research Institute for Astrophysics and Astronomy of Maragha (RIAAM), P.O. Box 55134-441, Maragha, Iran \\ ${ }^{6}$ Department of Physics, California State University Fullerton, 800 North State College Boulevard, Fullerton, CA 92831, USA \\ ${ }^{7}$ Department of Mathematics, Boise State University, 1910 University Drive, Boise, ID 83725, USA
}

Correspondence should be addressed to C. Corda; cordac.galilei@gmail.com

Received 8 December 2013; Accepted 21 December 2013; Published 11 February 2014

Academic Editor: Xiaoxiong Zeng

Copyright (c) 2014 C. Corda et al. This is an open access article distributed under the Creative Commons Attribution License, which permits unrestricted use, distribution, and reproduction in any medium, provided the original work is properly cited. The publication of this article was funded by $\mathrm{SCOAP}^{3}$.

It is known that the nonstrictly thermal character of the Hawking radiation spectrum harmonizes Hawking radiation with black hole (BH) quasi-normal modes (QNM). This paramount issue has been recently analyzed in the framework of both Schwarzschild BHs $(\mathrm{SBH})$ and Kerr BHs $(\mathrm{KBH})$. In this assignment, we generalize the analysis to the framework of nonextremal Reissner-Nordström BHs (RNBH). Such a generalization is important because in both Schwarzschild and Kerr BHs an absorbed (emitted) particle has only mass. Instead, in RNBH the particle has charge as well as mass. In doing so, we expose that, for the RNBH, QNMs can be naturally interpreted in terms of quantum levels for both particle emission and absorption. Conjointly, we generalize some concepts concerning the RNBH's "effective states."

\section{Introduction}

A RNBH of mass $M$ is identical to a SBH of mass $M$ except that a RNBH has the nonzero charge quantity $Q$. In this paper, we are interested in RNBHs with the nonextremal constraint $M>Q$ [1]. The quantity $Q$ is the physical mechanism for the RNBH's dual horizons from (1) in [1]:

$$
r_{ \pm}=R_{ \pm_{\mathrm{RNBH}}}(M, Q)=M \pm \sqrt{M^{2}-Q^{2}}
$$

because the RNBH outer (event) horizon radius $R_{+_{\mathrm{RNBH}}}(M, Q)$ and the $R N B H$ inner (Cauchy) horizon radius $R_{-{ }_{\mathrm{RNBH}}}(M, Q)$ are clearly functions of both $M$ and $Q$, not just $M$, as in the well known case of the $S B H$ horizon radius

$$
r_{s}=R_{\mathrm{SBH}}(M)=2 M .
$$

Energy conservation plays a fundamental role in $\mathrm{BH}$ radiance [2] because the emission or absorption of Hawking quanta with mass $m$ and energy-frequency $\omega$ causes a $\mathrm{BH}$ of mass $M$ to undergo a transition between discrete energy spectrum levels [3-7], where

$$
E=m=\omega=\Delta M
$$

for $G=c=k_{B}=\hbar=1 / 4 \pi \epsilon_{0}=1$ (Planck units). Given that emission and absorption are reverse processes for the quantized energy spectrum conservation [3-7], we consider this pair of transitions as being equal in magnitude but opposite in direction from the neutral radius perspective of $r_{0}=\left(r_{+}+r_{-}\right) / 2$.

It is known that the countable character of successive emissions of Hawking quanta which is a consequence of 
the nonstrictly thermal character of the Hawking radiation spectrum (see [3-12]) generates a natural correspondence between Hawking radiation and BH QNMs [3-7]. Moreover, it has also been shown that QNMs can be naturally interpreted in terms of quantum levels, where the emission or absorption of a particle is interpreted as a transition between two distinct levels on the discrete energy spectrum [3-7]. The thermal spectrum correction is an imperative adjustment to the physical interpretation of BH QNMs because these results are important to realize the underlying unitary quantum gravity theory [3-7]. Hod's intriguing works [13, 14] suggested that $\mathrm{BH}$ QNMs carry principle information regarding a BH's horizon area quantization. Hod's influential conjecture was later refined and clarified by Maggiore [15]. Moreover, it is also believed that QNMs delve into the microstructure of spacetime [16].

To make sense of the state space for the energy spectrum states and the underlying $\mathrm{BH}$ perturbation field states, an effective framework based on the nonstrictly thermal behavior of Hawking's framework began to emerge [3-7]. In the midst of this superceding $\mathrm{BH}$ effective framework [3-7], the $\mathrm{BH}$ effective state concept was originally introduced for $\mathrm{KBHs}$ in [6] and subsequently applied through Hawking's periodicity arguments $[17,18]$ to the $\mathrm{BH}$ tunneling mechanism's nonstrictly black body spectrum [7]. The effective state is meaningful to $\mathrm{BH}$ physics and thermodynamics research because one needs additional features and knowledge to consider in future experiments and observations.

In this paper, our objective is to apply the nonstrictly thermal $\mathrm{BH}$ effective framework of [3-7] to nonextremal RNBHs. Thus, upon recalling that a $\mathrm{RNBH}$ of mass $M$ is identical to a $\mathrm{SBH}$ of mass $M$ except that a $\mathrm{RNBH}$ has the charge $Q$, we prepare for our $\mathrm{BH} \mathrm{QNM}$ investigation by reviewing relevant portions of the $\mathrm{SBH}$ effective framework [3-7] for quantities related to $\mathrm{SBH}$ states and transitions in Section 2. Then in Section 3, we launch our RNBH QNM exploration by introducing a $\mathrm{RNBH}$ effective framework for quantities pertaining to RNBH states and transitions. Finally, we conclude with a brief comparison between the fundamental SBH and RNBH results in Section 4 followed by the recapitulation in Section 5.

\section{Schwarzschild Black Hole Framework: Background and Review}

2.1. Schwarzschild Black Hole States and Transitions. Here, we recall some quantities that characterize the $\mathrm{SBH}$.

First, consider a SBH of initial mass $M$, when the $\mathrm{SBH}$ emits or absorbs a quantum of energy-frequency $\omega$ (for particle mass $m$ and SBH mass change $\Delta M$, such that $m=\omega=$ $\Delta M$ ) to achieve a final mass of $M-\omega$ or $M+\omega$, respectively, for the SBH mass-energy transition between states in state space. Thus, we follow [3-5], where the SBH initial and final horizon area are

$$
\begin{gathered}
A_{\mathrm{SBH}}(M)=16 \pi M^{2}=4 \pi R_{\mathrm{SBH}}^{2}(M), \\
A_{\mathrm{SBH}}(M \pm \omega)=16 \pi(M \pm \omega)^{2}=4 \pi R_{\mathrm{SBH}}^{2}(M \pm \omega),
\end{gathered}
$$

respectively, for the $S B H$ area quanta number

$$
N_{\mathrm{SBH}}(M, \omega)=\frac{A_{\mathrm{SBH}}(M)}{\left|\Delta A_{\mathrm{SBH}}(M, \omega)\right|},
$$

such that the SBH horizon area change for the corresponding mass change $\triangle M$ is

$$
\begin{aligned}
\Delta A_{\mathrm{SBH}}(M, \omega) & =A_{\mathrm{SBH}}(M \pm \omega)-A_{\mathrm{SBH}}(M) \\
& =32 \pi M \omega+O\left(\omega^{2}\right) \sim 32 \pi M \Delta M \\
& =32 \pi M \Delta E,
\end{aligned}
$$

because the transition's minus $(-)$ and plus $(+)$ signs depend on emission and absorption, respectively. Next, in [3-5], the Bekenstein-Hawking SBH initial and final entropy are

$$
\begin{gathered}
S_{\mathrm{SBH}}(M)=\frac{A_{\mathrm{SBH}}(M)}{4}, \\
S_{\mathrm{SBH}}(M \pm \omega)=\frac{A_{\mathrm{SBH}}(M \pm \omega)}{4},
\end{gathered}
$$

respectively, where the corresponding SBH entropy change is

$$
\Delta S_{\mathrm{SBH}}(M, \omega)=\frac{\Delta A_{\mathrm{SBH}}(M, \omega)}{4} .
$$

Subsequently, the SBH initial and final total entropy are [3-5]

$$
\begin{aligned}
S_{\mathrm{SBH}-\text { total }}(M)= & S_{\mathrm{SBH}}(M)-\ln S_{\mathrm{SBH}}(M) \\
& +\frac{3}{2 A_{\mathrm{SBH}}(M)}, \\
S_{\mathrm{SBH}-\text { total }}(M \pm \omega)= & S_{\mathrm{SBH}}(M \pm \omega)-\ln S_{\mathrm{SBH}}(M \pm \omega) \\
& +\frac{3}{2 A_{\mathrm{SBH}}(M \pm \omega)},
\end{aligned}
$$

respectively. Additionally, the $S B H$ initial and final Hawking temperature are [3-5]

$$
\begin{gathered}
T_{H_{\mathrm{SBH}}}(M)=\frac{1}{8 \pi M}, \\
T_{H_{\mathrm{SBH}}}(M \pm \omega)=\frac{1}{8 \pi(M \pm \omega)},
\end{gathered}
$$

respectively. Therefore, the quantum transition's SBH emission tunneling rate is [3-5]

$$
\begin{aligned}
\Gamma_{\mathrm{SBH}}(M, \omega) & \sim \exp \left[-8 \pi M \omega\left(1-\frac{\omega}{2 M}\right)\right] \\
& \sim \exp \left[-\frac{\omega}{T_{H_{\mathrm{SBH}}}(M)}\left(1-\frac{\omega}{R_{\mathrm{SBH}}(M)}\right)\right] \\
& \sim \exp \left[+\Delta S_{\mathrm{SBH}}(M, \omega)\right] .
\end{aligned}
$$


2.2. Schwarzschild Black Hole Effective States and Transitions. Here, we recall some effective quantities that characterize the SBH.

Given that $M$ is the mass state before and $M \pm \omega$ is the mass state after the quantum transition, the SBH effective mass and SBH effective horizon are, respectively, identified in [3-5] as

$$
\begin{gathered}
M_{E}(M, \omega)=\frac{M+(M \pm \omega)}{2}=M \pm \frac{\omega}{2} \\
R_{E_{\mathrm{SBH}}}(M, \omega)=2 M_{E}(M, \omega),
\end{gathered}
$$

which are average quantities between the two states before and after the process [3-5]. Consequently, using (4) and (12) we define the SBH effective horizon area as

$$
\begin{aligned}
A_{E_{\mathrm{SBH}}}(M, \omega) & \equiv \frac{A_{\mathrm{SBH}}(M)+A_{\mathrm{SBH}}(M \pm \omega)}{2} \\
& =16 \pi M_{E}^{2}(M, \omega)=4 \pi R_{E_{\mathrm{SBH}}}^{2}(M, \omega),
\end{aligned}
$$

which is the average of the SBH's initial and final horizon areas. Subsequently, utilizing (7), the Bekenstein-Hawking SBH effective entropy is defined as

$$
S_{E_{\mathrm{SBH}}}(M, \omega) \equiv \frac{S_{\mathrm{SBH}}(M)+S_{\mathrm{SBH}}(M \pm \omega)}{2},
$$

and consequently employs (13) and (14) to define the $S B H$ effective total entropy as

$$
\begin{aligned}
& S_{E_{\mathrm{SBH}-\text { total }}}(M, \omega) \\
& \quad \equiv S_{E_{\mathrm{SBH}}}(M, \omega)-\ln S_{E_{\mathrm{SBH}}}(M, \omega)+\frac{3}{2 A_{E_{\mathrm{SBH}}}(M, \omega)} .
\end{aligned}
$$

Thus, employing (3) and (10), the SBH effective temperature is [3-5]

$$
\begin{aligned}
T_{E_{\mathrm{SBH}}}(M, \omega) & =\left(\frac{T_{H_{\mathrm{SBH}}}^{-1}(M)+T_{H_{\mathrm{SBH}}}^{-1}(M \pm \omega)}{2}\right)^{-1} \\
& =\left(8 \pi\left[\frac{M+M \pm \omega}{2}\right]\right)^{-1} \\
& =\frac{1}{4 \pi(2 M \pm \omega)}=\frac{1}{8 \pi M_{E}(M, \omega)},
\end{aligned}
$$

which is the inverse of the average value of the inverses of the initial and final Hawking temperatures. Consequently, (16) lets one rewrite (11) to define the SBH effective emission tunneling rate (in the Boltzmann-like form) as [3-5]

$$
\begin{aligned}
\Gamma_{E_{\mathrm{SBH}}}(M, \omega) & \sim \exp \left[-\frac{\omega}{T_{E_{\mathrm{SBH}}}(M, \omega)}\right] \\
& =\exp \left[+\Delta S_{E_{\mathrm{SBH}}}(M, \omega)\right],
\end{aligned}
$$

such that (14) defines the SBH effective entropy change as

$$
\Delta S_{E_{\mathrm{SBH}}}(M, \omega)=S_{\mathrm{SBH}}(M \pm \omega)-S_{\mathrm{SBH}}(M)=\frac{\Delta A_{E_{\mathrm{SBH}}}(M, \omega)}{4}
$$

because the SBH effective horizon area change is

$$
\Delta A_{E_{\mathrm{SBH}}}(M, \omega)=16 \pi M_{E}(M, \omega) \omega
$$

and the SBH effective area quanta number is

$$
N_{E_{\mathrm{SBH}}}(M, \omega)=\frac{A_{E_{\mathrm{SBH}}}(M, \omega)}{\Delta A_{E_{\mathrm{SBH}}}(M, \omega)} .
$$

2.3. Effective Application of Quasi-Normal Modes to the Schwarzschild Black Hole. Here, we recall how the $\mathrm{SBH}$ perturbation field QNM states can be applied to the $\mathrm{SBH}$ effective framework.

The quasi-normal frequencies (QNFs) are typically labeled as $\omega_{n l}$, where $l$ is the angular momentum quantum number $[3-5,15,19]$. Thus, for each $l$, such that $l \geq 2$ for gravitational perturbations, there is a countable sequence of QNMs labeled by the overtone number $n$, which is a natural number $[3-5,15]$.

Now $\left|\omega_{n}\right|$ is the damped harmonic oscillator's proper frequency that is defined as $[3-5,15]$

$$
\left|\omega_{n}\right|=\left(\omega_{0}\right)_{n}=\sqrt{\omega_{n_{\mathbb{R}}}^{2}+\omega_{n_{\sharp}}^{2}} .
$$

Maggiore [15] articulated that the establishment $\left|\omega_{n}\right|=\omega_{n_{R}}$ is only correct for the very long-lived and lowly excited QNMs approximation $\left|\omega_{n}\right| \gg \omega_{n}$, whereas for a lot of BH QNMs, such as those that are highly excited, the opposite limit is correct $[3-5,15]$. Therefore, the $\omega$ parameter in $(12)-(20)$ is substituted for the $\left|\omega_{n}\right|$ parameter [3-5] because we wish to employ BH QNFs. When $n$ is large, the SBH QNFs become independent of $l$ and thereby exhibit the nonstrictly thermal structure [3-5]

$$
\begin{aligned}
\omega_{n}= & \ln 3 \times T_{E_{\mathrm{SBH}}}\left(M,\left|\omega_{n}\right|\right)+2 \pi i\left(n+\frac{1}{2}\right) \times T_{E_{\mathrm{SBH}}}\left(M,\left|\omega_{n}\right|\right) \\
& +\mathcal{O}\left(n^{-1 / 2}\right)=\frac{\ln 3}{4 \pi\left[2 M-\left|\omega_{n}\right|\right]}+\frac{2 \pi i}{4 \pi\left[2 M-\left|\omega_{n}\right|\right]} \\
& \times\left(n+\frac{1}{2}\right)+\mathcal{O}\left(n^{-1 / 2}\right)=\frac{\ln 3}{8 \pi M_{E}\left(M,\left|\omega_{n}\right|\right)} \\
& +\frac{2 \pi(n+1 / 2)}{8 \pi M_{E}\left(M,\left|\omega_{n}\right|\right)} i+\mathcal{O}\left(n^{-1 / 2}\right),
\end{aligned}
$$

where

$$
\begin{gathered}
m_{n} \equiv \omega_{n_{\mathbb{R}}}=\frac{\ln 3}{8 \pi M_{E}\left(M,\left|\omega_{n}\right|\right)}, \\
p_{n} \equiv \omega_{n_{\|}}=\frac{2 \pi}{8 \pi M_{E}\left(M,\left|\omega_{n}\right|\right)}\left(n+\frac{1}{2}\right) .
\end{gathered}
$$


Thus, when referring to highly excited QNMs one gets $\left|\omega_{n}\right| \approx p_{n}$ [3-5], where the quantized levels differ from [15] because they are not equally spaced in exact form. Therefore, according to [3-5], we have

$$
\begin{aligned}
\left|\omega_{n}\right| & =\frac{\sqrt{(\ln 3)^{2}+4 \pi^{2}(n+1 / 2)^{2}}}{8 \pi M_{E}\left(M,\left|\omega_{n}\right|\right)} \\
& =T_{E_{\mathrm{SBH}}}\left(M,\left|\omega_{n}\right|\right) \sqrt{(\ln 3)^{2}+4 \pi^{2}\left(n+\frac{1}{2}\right)^{2}},
\end{aligned}
$$

which is solved to yield

$$
\left|\omega_{n}\right|=M-\sqrt{M^{2}-\frac{\sqrt{(\ln 3)^{2}+4 \pi^{2}(n+1 / 2)^{2}}}{4 \pi}}
$$

when we obey $\left|\omega_{n}\right|<M$ because a $\mathrm{BH}$ cannot emit more energy than its total mass.

\section{Reissner-Nordström Black Hole Framework: An Introduction}

We note that for this framework we consider the RNBH event horizon features, which are derived from the $R_{+_{\mathrm{RNBH}}}(M, Q)$ in (1).

3.1. Reissner-Nordström Black Hole States and Transitions. Here, we recall some quantities that characterize the RNBH.

First, consider a $\mathrm{RNBH}$ of initial mass $M$ and initial charge Q. Using (1), we define the $R N B H$ initial event horizon area as

$$
A_{+_{\mathrm{RNBH}}}(M, Q)=4 \pi\left(M+\sqrt{M^{2}-Q^{2}}\right)^{2}=4 \pi R_{+_{\mathrm{RNBH}}}^{2}(M, Q),
$$

the Bekenstein-Hawking RNBH initial entropy as

$$
S_{+_{\mathrm{RNBH}}}(M, Q)=\frac{A_{+_{\mathrm{RNBH}}}(M, Q)}{4},
$$

and the RNBH initial electrostatic potential as

$$
\Phi_{+}(M, Q)=\frac{Q}{4 \pi R_{+_{\mathrm{RNBH}}}(M, Q)}=\frac{Q}{4 \pi\left(M+\sqrt{M^{2}-Q^{2}}\right)} .
$$

Consequently, (17) of [2] identifies the RNBH initial Hawking temperature as

$$
\begin{aligned}
T_{+H_{\mathrm{RNBH}}}(M, Q) & =\frac{\sqrt{M^{2}-Q^{2}}}{2 \pi\left(M+\sqrt{M^{2}-Q^{2}}\right)^{2}} \\
& =\frac{R_{+_{\mathrm{RNBH}}}(M, Q)-R_{-{ }_{\mathrm{RNBH}}}(M, Q)}{A_{+_{\mathrm{RNBH}}}(M, Q)} .
\end{aligned}
$$

Second, consider when the RNBH emits or absorbs a quantum of energy-frequency $\omega$ with charge $q$ to achieve a final mass of $M-\omega$ or $M+\omega$ and a final charge of $Q-q$ or $Q+q$, respectively, for the $\mathrm{RNBH}$ mass-energy transition between states in state space. For this, all we need to do is replace the RNBH's mass and charge parameters in (26) and (29). Thus, (26) establishes the RNBH final event horizon area as

$$
\begin{aligned}
& A_{+_{\mathrm{RNBH}}}(M \pm \omega, Q \pm q) \\
& \quad=4 \pi R_{+_{\mathrm{RNBH}}}^{2}(M \pm \omega, Q \pm q) \\
& \quad=4 \pi\left((M \pm \omega)+\sqrt{(M \pm \omega)^{2}-(Q \pm q)^{2}}\right)^{2} .
\end{aligned}
$$

Equation (27) presents the Bekenstein-Hawking RNBH final entropy as

$$
S_{+_{\mathrm{RNBH}}}(M \pm \omega, Q \pm q)=\frac{A_{+_{\mathrm{RNBH}}}(M \pm \omega, Q \pm q)}{4},
$$

and (28) defines the RNBH final electrostatic potential as

$$
\begin{aligned}
\Phi_{+}(M \pm \omega, Q \pm q) \\
=\frac{Q}{4 \pi R_{+_{\mathrm{RNBH}}}(M \pm \omega, Q \pm q)} \\
=\frac{Q}{4 \pi\left((M \pm \omega)+\sqrt{(M \pm \omega)^{2}-(Q \pm q)^{2}}\right)}
\end{aligned}
$$

for usage in (29) of [20], where it is proposed that the $R N B H$ adiabatic invariant is

$$
\begin{aligned}
I_{+_{\mathrm{RNBH}}}(M, \omega, Q, q) & =\int \frac{\omega-\Phi_{+}(M \pm \omega, Q) q}{\omega} \\
& =\int \frac{\Delta M-\Phi_{+}(M \pm \Delta M, Q) \Delta Q}{\Delta M}
\end{aligned}
$$

because $\Delta Q=q$. Hence, (29) identifies the $R N B H$ final Hawking temperature as

$$
\begin{aligned}
T_{+} H_{\mathrm{RNBH}} & (M \pm \omega, Q \pm q) \\
= & \frac{R_{+_{\mathrm{RNBH}}}(M \pm \omega, Q \pm q)-R_{-_{\mathrm{RNBH}}}(M \pm \omega, Q \pm q)}{A_{+_{\mathrm{RNBH}}}(M \pm \omega, Q \pm q)} \\
= & \frac{\sqrt{(M \pm \omega)^{2}-(Q \pm q)^{2}}}{2 \pi\left((M \pm \omega)+\sqrt{(M \pm \omega)^{2}-(Q \pm q)^{2}}\right)^{2}} .
\end{aligned}
$$

Next, upon generalizing (16) in [2] and the work [21], we define the RNBH tunneling rate as

$$
\begin{aligned}
& \Gamma_{+_{\mathrm{RNBH}}}(M, \omega, Q, q) \\
& \sim \exp \left[-4 \pi\left(2 \omega\left(M \pm \frac{\omega}{2}\right)\right.\right. \\
& -(M \pm \omega) \sqrt{(M \pm \omega)^{2}-(Q \pm q)^{2}} \\
& \left.\left.+M \sqrt{M^{2}-Q^{2}}\right)\right] \\
& \sim \exp \left[\Delta S_{+_{\mathrm{RNBH}}}(M, \omega, Q, q)\right],
\end{aligned}
$$


where we utilize (30) to define the Bekenstein-Hawking RNBH entropy change as

$$
\Delta S_{+_{\mathrm{RNBH}}}(M, \omega, Q, q)=\frac{\Delta A_{+_{\mathrm{RNBH}}}(M, \omega, Q, q)}{4},
$$

such that the RNBH event horizon area change is

$$
\begin{aligned}
\Delta A_{+_{\mathrm{RNBH}}}(M, \omega, Q, q)= & A_{+_{\mathrm{RNBH}}}(M \pm \omega, Q \pm q) \\
& -A_{+_{\mathrm{RNBH}}}(M, Q)
\end{aligned}
$$

so we can define the RNBH event horizon area quanta number as

$$
N_{+_{\mathrm{RNBH}}}(M, \omega, Q, q)=\frac{A_{+_{\mathrm{RNBH}}}(M, Q)}{\left|\Delta A_{+_{\mathrm{RNBH}}}(M, \omega, Q, q)\right|} .
$$

3.2. Reissner-Nordström Black Hole Effective States and Transitions. Here, we define some effective quantities that characterize the RNBH.

The RNBH effective mass is equivalent to the SBH effective mass component of (12), which is

$$
M_{E}(M, \omega) \equiv \frac{M+(M \pm \omega)}{2}
$$

Next, we define the RNBH effective charge as

$$
Q_{E}(Q, q) \equiv \frac{Q+(Q \pm q)}{2}
$$

which is the average of the RNBH's initial charge $Q$ and final charge $Q \pm q$. From this, (1), (39), and (40) are used to define the corresponding $R N B H$ effective event horizon and $R N B H$ effective Cauchy horizon as

$$
\begin{aligned}
r_{ \pm E} \equiv & R_{ \pm E_{\mathrm{RNBH}}}(M, \omega, Q, q) \equiv M_{E}(M, \omega) \\
& \pm \sqrt{M_{E}^{2}(M, \omega)-Q_{E}^{2}(Q, q)}
\end{aligned}
$$

with respect to the energy conservation and pair production neutrality of (39). Next, we employ (26), (39), and (41) to define the RNBH effective event horizon area as

$$
\begin{aligned}
A_{+E_{\mathrm{RNBH}}} & (M, \omega, Q, q) \\
\equiv & 4 \pi R_{+E_{\mathrm{RNBH}}}^{2}(M, \omega, Q, q) \\
& \equiv 4 \pi\left(M_{E}(M, \omega)+\sqrt{M_{E}^{2}(M, \omega)-Q_{E}^{2}(Q, q)}\right)^{2},
\end{aligned}
$$

which is then used to define the RNBH effective entropy as

$$
S_{+E_{\mathrm{RNBH}}}(M, \omega, Q, q) \equiv \frac{A_{+E_{\mathrm{RNBH}}}(M, \omega, Q, q)}{4} .
$$

Afterwards, we use (28) and (42) to define the RNBH effective electrostatic potential as

$$
\begin{aligned}
\Phi_{+E}(M, \omega, Q, q) \\
\quad \equiv \frac{Q_{E}(Q, q)}{4 \pi R_{+E_{\mathrm{RNBH}}}(M, \omega, Q, q)} \\
\equiv \frac{Q_{E}(Q, q)}{4 \pi\left(M_{E}(M, \omega)+\sqrt{M_{E}^{2}(M, \omega)-Q_{E}^{2}(Q, q)}\right)}
\end{aligned}
$$

so we can utilize the $T_{E_{\mathrm{SBH}}}(M, \omega)$ in (16) along with (39), (40), and (44) to define the RNBH effective adiabatic invariant as

$$
\begin{aligned}
& I_{+E_{\mathrm{RNBH}}}(M, \omega, Q, q) \\
& \quad \equiv \int \frac{d M_{E}(M, \omega)-\Phi_{+E}(M, \omega, Q, q) d Q_{E}(Q, q)}{T_{E_{\mathrm{SBH}}}(M, \omega)} .
\end{aligned}
$$

At this point, (16) and (35) let us introduce and define the $R N B H$ effective temperature as

$$
\begin{aligned}
T_{+E_{\mathrm{RNBH}}} & (M, \omega, Q, q) \\
\equiv & \frac{\sqrt{(M \pm \omega / 2)^{2}-(Q \pm q / 2)^{2}}}{2 \pi\left[(M \pm \omega / 2)+\sqrt{(M \pm \omega / 2)^{2}-(Q \pm q / 2)^{2}}\right]^{2}} \\
\equiv & \frac{\sqrt{M_{E}^{2}(M, \omega)-Q_{E}^{2}(Q, q)}}{2 \pi\left(M_{E}(M, \omega)+\sqrt{M_{E}^{2}(M, \omega)-Q_{E}^{2}(Q, q)}\right)^{2}} \\
\equiv & \frac{R_{+E_{\mathrm{RNBH}}}(M, \omega, Q, q)-R_{-E_{\mathrm{RNBH}}}(M, \omega, Q, q)}{A_{+E_{\mathrm{RNBH}}}(M, \omega, Q, q)},
\end{aligned}
$$

which authorizes us to exercise (36) and (46) to rewrite (35) to define the $\mathrm{RNBH}$ effective tunneling rate as

$$
\begin{aligned}
& \Gamma_{+E_{\mathrm{RNBH}}}(M, \omega, Q, q) \sim \exp \left[\frac{ \pm \omega}{T_{+E_{\mathrm{RNBH}}}(M, \omega, Q, q)}\right] \\
& \quad \sim \exp \left[\Delta S_{+_{\mathrm{RNBH}}}(M, \omega, Q, q)\right],
\end{aligned}
$$

such that the RNBH effective entropy change is defined as

$$
\Delta S_{+E_{\mathrm{RNBH}}}(M, \omega, Q, q) \equiv \frac{\Delta A_{+E_{\mathrm{RNBH}}}(M, \omega, Q, q)}{4}
$$

for the RNBH effective event horizon area change

$$
\Delta A_{+E_{\mathrm{RNBH}}}(M, \omega, Q, q) \equiv \frac{2 \omega q+Q^{3} \pi}{\left(M^{2}-Q^{2}\right)^{3 / 2}}
$$

and the RNBH effective event horizon area quanta number

$$
N_{+E_{\mathrm{RNBH}}}(M, \omega, Q, q) \equiv \frac{A_{+E_{\mathrm{RNBH}}}(M, \omega, Q, q)}{\left|\Delta A_{+E_{\mathrm{RNBH}}}(M, \omega, Q, q)\right|} .
$$




\section{Effective Application of Quasi-Normal Modes to the Reissner-Nordström Black Hole}

Here, we explain how the RNBH perturbation field QNM states can be applied to the RNBH effective framework.

Similarly to SBH QNFs, the RNBH QNFs become independent of $l$ for large $n$ [22]. Thus, for large $n$, we have two families of the QNM:

$$
\begin{aligned}
\omega_{n}= & \ln 3 \times T_{+H_{\mathrm{SBH}}}(M, Q)-2 \pi\left(n+\frac{1}{2}\right) i \times T_{+H_{\mathrm{SBH}}}(M, Q) \\
& +\frac{q Q}{R_{+_{\mathrm{SBH}}}(M, Q)}
\end{aligned}
$$

$$
\begin{aligned}
\omega_{n}= & \ln 2 \times T_{+H_{\mathrm{RNBH}}}(M, Q)-2 \pi\left(n+\frac{1}{2}\right) i \times T_{+H_{\mathrm{RNBH}}}(M, Q) \\
& +\frac{q Q}{R_{+_{\mathrm{RNBH}}}(M, Q)}=\frac{\ln 2 \sqrt{M^{2}-Q^{2}}}{2 \pi\left(M+\sqrt{M^{2}-Q^{2}}\right)^{2}} \\
& -\frac{(n+1 / 2) \sqrt{M^{2}-Q^{2}}}{\left(M+\sqrt{M^{2}-Q^{2}}\right)^{2}} i+\frac{q Q}{R_{+_{\mathrm{RNBH}}}(M, Q)} .
\end{aligned}
$$

Now the approximation of (51) and (52) is only relevant under the assumption that the $\mathrm{BH}$ radiation spectrum is strictly thermal [3-5] because they both use the Hawking temperature $T_{+H_{\mathrm{RNB}}}$ in (29). Hence, to operate in compliance with [3-5] and thereby account for the thermal spectrum deviation of (35), we opt to select the (52) case and upgrade it by effectively replacing its $T_{H_{\mathrm{RNBH}}}$ in (29) with the $T_{+E_{\mathrm{RNBH}}}$ in (46). Therefore, the corrected expression for the RNBH QNFs of (52) which encodes the nonstrictly thermal behavior of the radiation spectrum is defined as

$$
\begin{aligned}
\omega_{n} \equiv & \ln 2 \times T_{+E_{\mathrm{RNBH}}}\left(M,\left|\omega_{n}\right|, Q, q\right) \\
& -2 \pi\left(n+\frac{1}{2}\right) i \times T_{+E_{\mathrm{RNBH}}}\left(M,\left|\omega_{n}\right|, Q, q\right) \\
& +\frac{q Q_{E}(Q, q)}{R_{+E_{\mathrm{RNBH}}}\left(M,\left|\omega_{n}\right|, Q, q\right)} \\
\equiv & \frac{\ln 2 \sqrt{M_{E}^{2}\left(M,\left|\omega_{n}\right|\right)-Q_{E}^{2}(Q, q)}}{2 \pi\left(M_{E}\left(M,\left|\omega_{n}\right|\right)+\sqrt{M_{E}^{2}\left(M,\left|\omega_{n}\right|\right)-Q_{E}^{2}(Q, q)}\right)^{2}} \\
& -\frac{(n+1 / 2) \sqrt{M_{E}^{2}\left(M,\left|\omega_{n}\right|\right)-Q_{E}^{2}(Q, q)}}{\left(M_{E}\left(M,\left|\omega_{n}\right|\right)+\sqrt{M_{E}^{2}\left(M,\left|\omega_{n}\right|\right)-Q_{E}^{2}(Q, q)}\right)^{2}} i \\
& +\frac{q Q_{E}(Q, q)}{R_{+E_{\mathrm{RNBH}}}\left(M,\left|\omega_{n}\right|, Q, q\right)} .
\end{aligned}
$$

From (39), (41), and (46) we define the effective quantities associated with the QNMs as

$$
\begin{aligned}
& M_{E}\left(M,\left|\omega_{n}\right|\right) \equiv \frac{M+\left(M-\left|\omega_{n}\right|\right)}{2}, \\
& r_{ \pm E} \equiv R_{ \pm E_{\mathrm{RNBH}}}\left(M,\left|\omega_{n}\right|, Q, q\right)= \\
& =M_{E}\left(M,\left|\omega_{n}\right|\right) \pm \sqrt{M_{E}^{2}\left(M,\left|\omega_{n}\right|\right)-Q_{E}^{2}(Q, q)}, \\
& T_{+E_{\mathrm{RNBH}}}\left(M,\left|\omega_{n}\right|, Q, q\right) \\
& \equiv \frac{\sqrt{\left(M-\left|\omega_{n}\right| / 2\right)^{2}-(Q-q / 2)^{2}}}{2 \pi\left[\left(M-\left|\omega_{n}\right| / 2\right)+\sqrt{\left(M-\left|\omega_{n}\right| / 2\right)^{2}-(Q-q / 2)^{2}}\right]^{2}} \\
& =\frac{\sqrt{M_{E}^{2}\left(M,\left|\omega_{n}\right|\right)-Q_{E}^{2}(Q, q)}}{2 \pi\left(M_{E}\left(M,\left|\omega_{n}\right|\right)+\sqrt{M_{E}^{2}\left(M,\left|\omega_{n}\right|\right)-Q_{E}^{2}(Q, q)}\right)^{2}} \\
& =\frac{R_{+E_{\mathrm{RNBH}}}\left(M,\left|\omega_{n}\right|, Q, q\right)-R_{-E_{\mathrm{RNBH}}}\left(M,\left|\omega_{n}\right|, Q, q\right)}{A_{+E_{\mathrm{RNBH}}}\left(M,\left|\omega_{n}\right|, Q, q\right)},
\end{aligned}
$$

respectively, for the quantum overtone number $n$ in (53). Hence, (53) lets us rewrite the $\mathrm{SBH}$ case of (23) to present the RNBH case

$$
\begin{aligned}
m_{n} \equiv & \ln 2 \times T_{+E_{\mathrm{RNBH}}}\left(M,\left|\omega_{n}\right|, Q, q\right) \\
& +\frac{e Q_{E}(Q, q)}{R_{+E_{\mathrm{RNBH}}}\left(M,\left|\omega_{n}\right|, Q, q\right)} \\
= & \frac{\ln 2 \sqrt{M_{E}^{2}\left(M,\left|\omega_{n}\right|\right)-Q_{E}^{2}(Q, q)}}{2 \pi\left(M_{E}\left(M,\left|\omega_{n}\right|\right)+\sqrt{M_{E}^{2}\left(M,\left|\omega_{n}\right|\right)-Q_{E}^{2}(Q, q)}\right)^{2}} \\
& +\frac{q Q_{E}(Q, q)}{R_{+E_{\mathrm{RNBH}}}\left(M,\left|\omega_{n}\right|, Q, q\right)}, \\
p_{n} \equiv & -2 \pi\left(n+\frac{1}{2}\right) \times T_{+E_{\mathrm{RNBH}}}\left(M,\left|\omega_{n}\right|, Q, q\right) \\
= & -\frac{(n+1 / 2) \sqrt{M_{E}^{2}\left(M,\left|\omega_{n}\right|\right)-Q}}{\left(M_{E}\left(M,\left|\omega_{n}\right|\right)+\sqrt{M_{E}^{2}\left(M,\left|\omega_{n}\right|\right)-Q_{E}^{2}(Q, q)}\right)^{2}} .
\end{aligned}
$$


Thus, we recall that if $\left|\omega_{n}\right| \approx p_{n}$, then we are referring to highly excited QNMs [3-5]. Therefore, the SBH case of (24) becomes the RNBH case

$$
\begin{aligned}
& \left|\omega_{n}\right| \\
& \equiv \frac{\sqrt{M_{E}^{2}\left(M,\left|\omega_{n}\right|\right)-Q_{E}^{2}(Q, q)} \sqrt{(\ln 2)^{2}-4 \pi^{2}(n+1 / 2)^{2}}}{2 \pi\left(M_{E}\left(M,\left|\omega_{n}\right|\right)+\sqrt{M_{E}^{2}\left(M,\left|\omega_{n}\right|\right)-Q_{E}^{2}(Q, q)}\right)^{2}} \\
& \quad+\frac{q Q_{E}(Q, q)}{R_{+E_{\mathrm{RNBH}}}\left(M,\left|\omega_{n}\right|, Q, q\right)}=T_{+E_{\mathrm{RNBH}}}\left(M,\left|\omega_{n}\right|, Q, q\right) \\
& \quad \times \sqrt{(\ln 2)^{2}-4 \pi^{2}\left(n+\frac{1}{2}\right)^{2}}+\frac{q Q_{E}(Q, q)}{R_{+E_{\mathrm{RNBH}}}\left(M,\left|\omega_{n}\right|, Q, q\right)} .
\end{aligned}
$$

Hence, upon considering (40) and (54), one can rewrite (58) as

$$
\begin{aligned}
\left|\omega_{n}\right| \equiv & \frac{\sqrt{\left(M-\left|\omega_{n}\right| / 2\right)^{2}-(\mathrm{Q}-q / 2)^{2}} \sqrt{(\ln 2)^{2}-4 \pi^{2}(n+1 / 2)^{2}}}{2 \pi\left[\left(M-\left|\omega_{n}\right| / 2\right)+\sqrt{\left(M-\left|\omega_{n}\right| / 2\right)^{2}-(\mathrm{Q}-q / 2)^{2}}\right]^{2}} \\
& +\frac{q(\mathrm{Q}-q / 2)}{\left(M-\left|\omega_{n}\right| / 2\right)+\sqrt{\left(M-\left|\omega_{n}\right| / 2\right)^{2}-(\mathrm{Q}-q / 2)^{2}}},
\end{aligned}
$$

where the solution of (59) in terms of $\left|\omega_{n}\right|$ will be the answer of $\left|\omega_{n}\right|$. Therefore, given a quantum transition between the levels $n$ and $n-1$, we define $\left|\Delta \omega_{n, n-1}\right| \equiv\left|\omega_{n}-\omega_{n-1}\right|$ where (41)-(45) are rewritten as

$$
\begin{gathered}
r_{ \pm E} \equiv R_{ \pm E_{\mathrm{RNBH}}}\left(M,\left|\Delta \omega_{n, n-1}\right|, Q, q\right) \\
\equiv M_{E}\left(M,\left|\Delta \omega_{n, n-1}\right|\right) \\
\quad \pm \sqrt{M_{E}^{2}\left(M,\left|\Delta \omega_{n, n-1}\right|\right)-Q_{E}^{2}(Q, q)}, \\
A_{+E_{\mathrm{RNBH}}}\left(M,\left|\Delta \omega_{n, n-1}\right|, Q, q\right) \\
\equiv 4 \pi R_{+E_{\mathrm{RNBH}}}^{2}\left(M,\left|\Delta \omega_{n, n-1}\right|, Q, q\right) \\
\equiv 4 \pi\left(M_{E}\left(M,\left|\Delta \omega_{n, n-1}\right|\right)\right. \\
\left.\quad+\sqrt{M_{E}^{2}\left(M,\left|\Delta \omega_{n, n-1}\right|\right)-Q_{E}^{2}(Q, q)}\right)^{2},
\end{gathered}
$$

$$
\begin{aligned}
& S_{+E_{\mathrm{RNBH}}}\left(M,\left|\Delta \omega_{n, n-1}\right|, Q, q\right) \equiv \frac{A_{+E_{\mathrm{RNBH}}}\left(M,\left|\Delta \omega_{n, n-1}\right|, Q, q\right)}{4}, \\
& \Phi_{+E}\left(M,\left|\Delta \omega_{n, n-1}\right|, Q, q\right) \\
& \equiv \frac{Q_{E}(Q, q)}{4 \pi R_{+E_{\mathrm{RNBH}}}\left(M,\left|\Delta \omega_{n, n-1}\right|, Q, q\right)} \\
& \equiv \frac{Q_{E}(Q, q)}{4 \pi\left(M_{E}\left(M,\left|\Delta \omega_{n, n-1}\right|\right)+\sqrt{M_{E}^{2}\left(M,\left|\Delta \omega_{n, n-1}\right|\right)-Q_{E}^{2}(\mathrm{Q}, q)}\right)}, \\
& I_{+E_{\mathrm{RNBH}}}\left(M,\left|\Delta \omega_{n, n-1}\right|, Q, q\right) \\
& =\int \frac{d M_{E}\left(M,\left|\Delta \omega_{n, n-1}\right|\right)-\Phi_{+E}\left(M,\left|\Delta \omega_{n, n-1}\right|, Q, q\right) d Q_{E}(Q, q)}{T_{E_{\mathrm{SBH}}}\left(M,\left|\Delta \omega_{n, n-1}\right|\right)},
\end{aligned}
$$

and (47)-(50) become

$$
\begin{gathered}
\Gamma_{+E_{\mathrm{RNBH}}}\left(M,\left|\Delta \omega_{n, n-1}\right|, Q, q\right) \\
\sim \exp \left[\frac{ \pm\left|\Delta \omega_{n, n-1}\right|}{T_{+E_{\mathrm{RNBH}}}\left(M,\left|\Delta \omega_{n, n-1}\right|, Q, q\right)}\right] \\
\sim \exp \left[\Delta S_{+_{\mathrm{RNBH}}}\left(M,\left|\Delta \omega_{n, n-1}\right|, Q, q\right)\right], \\
\Delta S_{+E_{\mathrm{RNBH}}}\left(M,\left|\Delta \omega_{n, n-1}\right|, Q, q\right) \\
\equiv \frac{\Delta A_{+E_{\mathrm{RNBH}}}\left(M,\left|\Delta \omega_{n, n-1}\right|, Q, q\right)}{4}, \\
\Delta A_{+E_{\mathrm{RNBH}}}\left(M,\left|\Delta \omega_{n, n-1}\right|, Q, q\right) \equiv \frac{2\left|\Delta \omega_{n, n-1}\right| q+\pi Q^{3}}{\left(M^{2}-Q^{2}\right)^{3 / 2}}, \\
N_{+E_{\mathrm{RNBH}}}\left(M,\left|\Delta \omega_{n, n-1}\right|, Q, q\right) \\
\equiv \frac{A_{+E_{\mathrm{RNBH}}}\left(M,\left|\Delta \omega_{n, n-1}\right|, Q, q\right)}{\left|\Delta A_{+E_{\mathrm{RNBH}}}\left(M,\left|\Delta \omega_{n, n-1}\right|, Q, q\right)\right|}
\end{gathered}
$$

respectively.

\section{A Brief Comparison}

Here, we will show that the SBH results of Section 2 are in fundamental agreement with the RNBH results of Section 3 for small $Q$, where we recall that the RNBH of mass $M$ is identical to a SBH of mass $M$ except that a $\mathrm{RNBH}$ has the nonzero charge quantity $Q$.

First, for small $Q$, the SBH's $T_{E_{\mathrm{SBH}}}(M, \omega)$ of $(16)$ is related to the RNBH's $T_{+E_{\mathrm{RNBH}}}(M, \omega, Q, q)$ of $(46)$ as

$$
\begin{aligned}
& T_{+E_{\mathrm{RNBH}}}(M, \omega, Q, q) \\
& \quad \equiv T_{E_{\mathrm{SBH}}}(M, \omega)-\frac{3 q^{2} Q^{2}}{8(2 m \pm \omega)^{5} \pi}+\mathcal{O}\left(Q^{4}, q^{4}\right) .
\end{aligned}
$$


Second, for small $Q$, the SBH's $A_{E_{\mathrm{SBH}}}(M, \omega)$ of (13) complies with the RNBH's $A_{+E_{\mathrm{RNBH}}}(M, \omega, Q, q)$ of $(42)$ as

$$
A_{+E_{\mathrm{RNBH}}}(M, \omega, Q, q) \equiv A_{E_{\mathrm{SBH}}}(M, \omega)-8 \pi Q^{2}+\mathcal{O}\left(Q^{4}\right) .
$$

Third, for small $Q$, the SBH's $S_{E_{S B H}}(M, \omega)$ of (14) corresponds with the RNBH's $S_{+E_{\mathrm{RNBH}}}(M, \omega, Q, q)$ of $(43)$ as

$$
S_{+E_{\mathrm{RNBH}}}(M, \omega, Q, q) \equiv S_{E_{\mathrm{SBH}}}(M, \omega)-2 \pi Q^{2}+\mathcal{O}\left(Q^{4}\right) .
$$

Fourth, for small Q, the SBH's QNF $\left|\omega_{n}\right|$ of (24) is consistent with the RNBH's QNF $\left|\omega_{n}\right|$ of (58) and (59) as

$$
\begin{aligned}
\left|\omega_{n}\right| \equiv & \frac{\sqrt{\ln 2^{2}-4 \pi^{2}(n+1 / 2)^{2}}}{4\left(2 M-\left|\omega_{n}\right|\right) \pi}+\frac{q Q}{2 M-\left|\omega_{n}\right|} \\
= & \left(\left(3 \left(16 \pi M^{2}-16 \pi\left|\omega_{n}\right| M+4 \pi\left|\omega_{n}\right|^{2}\right.\right.\right. \\
& \quad+\sqrt{-(\ln 2+\pi+2 \pi n)(-\ln 2+\pi+2 \pi n)})) \\
& \left.\times\left(8\left(2 M-\left|\omega_{n}\right|\right)^{5} \pi\right)^{-1}\right) Q^{2} q^{2} \\
= & \frac{q^{2}}{4 M-\left|\omega_{n}\right|}+\mathcal{O}\left(Q^{4}, q^{4}\right)
\end{aligned}
$$

which can be applied to (62)-(63) by replacing the $\omega$ parameter with the pertinent $\left|\omega_{n}\right|$. Hence, (62)-(65) indicate that in general the $\mathrm{SBH}$ results of Section 2 are fundamentally consistent with the RNBH results of Section 3 for small $Q$. Moreover, in (65) for large $n$, the result is consistent with the $\mathrm{SBH}$ because $\ln 2$ is negligible, but for small $n$ there is an argument between scientists regarding $\ln 2$ and $\ln 3$ because these refer to the two distinct QNM families of (51) and (52).

Here, we provide the physical answer of (65) for the case of emission by using the fact that $Q$ is small, so the term which includes $Q^{2}$ is also very small and therefore negligible:

$$
\begin{aligned}
\left(\omega_{0}\right)_{n} \equiv & \left|\omega_{n}\right| \approx M \\
& -\sqrt{M^{2}+\frac{q^{2}}{2}-Q q-\frac{1}{4 \pi} \sqrt{\ln 2^{2}-4 \pi^{2}\left(n+\frac{1}{2}\right)^{2}}} .
\end{aligned}
$$

Thus, by setting $\left(\omega_{0}\right)_{n} \equiv\left|\omega_{n}\right|$ we obtain

$$
\begin{aligned}
\Delta M_{n} \equiv & -\Delta \omega_{n, n-1}=\left(\omega_{0}\right)_{n-1}-\left(\omega_{0}\right)_{n} \\
\equiv & \sqrt{M^{2}+\frac{q^{2}}{2}-Q q-\frac{1}{4 \pi} \sqrt{(\ln 2)^{2}+4 \pi^{2}\left(n+\frac{1}{2}\right)^{2}}} \\
& -\sqrt{M^{2}+\frac{q^{2}}{2}-Q q-\frac{1}{4 \pi} \sqrt{(\ln 2)^{2}+4 \pi^{2}\left(n-\frac{1}{2}\right)^{2}}}
\end{aligned}
$$

for an emission involving quantum levels $n$ and $n-1$, which becomes

$$
\begin{aligned}
\Delta M_{n} \approx & \sqrt{M^{2}+\frac{q^{2}}{2}-Q q-\frac{1}{2}\left(n+\frac{1}{2}\right)} \\
& -\sqrt{M^{2}+\frac{q^{2}}{2}-Q q-\frac{1}{2}\left(n-\frac{1}{2}\right)}
\end{aligned}
$$

for large $n$.

\section{Conclusion Remarks}

We began our paper by summarizing some basic similarities and differences between SBHs and RNBHs in terms of charge and horizon radii. Moreover, we briefly explored the ParikhWilczek statement that explains how energy conservation and pair production $[2,23]$ are fundamentally related to such $\mathrm{BH}$. For a BH's discrete energy spectrum, the emission or absorption of a particle yields a transition between two distinct levels, where particle emission and absorption are reverse processes [3-7]. For this, we touched on the important issue that the nonstrictly thermal character of Hawking's radiation spectrum generates a natural correspondence between Hawking's radiation and BH QNMs, because these structures exemplify features of the BH's energy spectrum [3-5], which has been recently generalized to the emerging concept of a BH's effective state $[6,7]$.

Next, we prepared for our nonextremal RNBH QNM investigation by first reviewing relevant portions of the $\mathrm{SBH}$ effective framework [3-5] in Section 2. There, we listed the noneffective and effective quantities for SBH states and transitions, with direct application to the QNM characterization and framework of [3-5]. Subsequently, in Section 3, we identified some existing noneffective quantities and introduced new effective quantities for RNBH states and transitions so we could apply the $\mathrm{BH}$ framework of [3-5] to implement a RNBH framework. These results are crucial because the effective quantities in [3-5] have been achieved for the stable four-dimensional RNBH solution in Einstein's general relativity-now effective frameworks exist for the $\mathrm{SBH}, \mathrm{KBH}$, and (nonextremal) RNBH solutions.

Ultimately, the RNBH effective quantities permitted us to utilize both the KBH's effective state concept $[6,7]$ and the $\mathrm{BH}$ QNMs [3-5] to construct a foundation for the RNBH's effective state in this developing $\mathrm{BH}$ effective framework. The RNBH effective state concept is meaningful because, as scientists who wish to demystify the $\mathrm{BH}$ paradigm, we need additional features and knowledge to consider in future experiments and observations.

Finally, we stress that the nonstrictly thermal behavior of the Hawking radiation spectrum has been recently used to construct two very intriguing proposals to solve the $\mathrm{BH}$ information loss paradox. The first one received the First Award in the 2013 Gravity Research Foundation Essay Competition [12]. The latter won the Community Rating at the 2013 FQXi Essay Contest-It from Bit or Bit from It [24]. We are working to extend this second approach to the RNBH framework [25]. 


\section{Conflict of Interests}

The authors declare that there is no conflict of interests regarding the publication of this paper.

\section{Acknowledgments}

S. H. Hendi wishes to thank the Shiraz University Research Council. The work of S. H. Hendi has been supported financially by the Research Institute for Astronomy and Astrophysics of Maragha (RIAAM), Iran.

\section{References}

[1] B. Wang, R. K. Su, P. K. N. Yu, and E. C. M. Young, "Can a nonextremal Reissner-Nordström black hole become extremal by assimilating an infalling charged particle and shell?" Physical Review D, vol. 57, no. 8, pp. 5284-5286, 1998.

[2] M. K. Parikh and F. Wilczek, "Hawking radiation as tunneling," Physical Review Letters, vol. 85, no. 24, pp. 5042-5045, 2000.

[3] C. Corda, "Effective temperature for black holes," Journal of High Energy Physics, vol. 2011, p. 101, 2011.

[4] C. Corda, "Black hole quantum spectrum," The European Physical Journal C, vol. 73, p. 2665, 2013.

[5] C. Corda, "Effective temperature, hawking radiation and quasinormal modes," International Journal of Modern Physics D, vol. 21, no. 11, Article ID 1242023, 2012.

[6] C. Corda, S. H. Hendi, R. Katebi, and N. O. Schmidt, "Effective state, Hawking radiation and quasi-normal modes for Kerr black holes," Journal of High Energy Physics, vol. 2013, p. 8, 2013.

[7] C. Corda, "Non-strictly black body spectrum from the tunnelling mechanism," Annals of Physics, vol. 337, pp. 49-54, 2013.

[8] B. Zhang, Q.-Y. Cai, L. You, and M. S. Zhan, "Hidden messenger revealed in Hawking radiation: a resolution to the paradox of black hole information loss," Physics Letters B, vol. 675, no. 1, pp. 98-101, 2009.

[9] B. Zhang, Q.-Y. Cai, M. S. Zhan, and L. You, "Entropy is conserved in Hawking radiation as tunneling: a revisit of the black hole information loss paradox," Annals of Physics, vol. 326, no. 2, pp. 350-363, 2011

[10] B. Zhang, Q.-Y. Cai, M. S. Zhan, and L. You, "Noncommutative information is revealed from Hawking radiation as tunneling," EPL (Europhysics Letters), vol. 94, no. 2, Article ID 20002, 2011.

[11] B. Zhang, Q.-Y. Cai, M. S. Zhan, and L. You, "Comment on 'What the information loss is not"' http://arxiv.org/abs/ 1210.2048. In press.

[12] B. Zhang, Q. Y. Cai, M. S. Zhan, and L. You, "Information conservation is fundamental: recovering the lost information in Hawking radiation," International Journal of Modern Physics D, vol. 22, Article ID 1341014, 8 pages, 2013.

[13] S. Hod, "Gravitation, the quantum and Bohr's correspondence principle," General Relativity and Gravitation, vol. 31, no. 11, pp. 1639-1644, 1999.

[14] S. Hod, "Bohr's correspondence principle and the area spectrum of quantum black holes," Physical Review Letters, vol. 81, no. 20, pp. 4293-4296, 1998.

[15] M. Maggiore, "Physical interpretation of the spectrum of black hole quasinormal modes," Physical Review Letters, vol. 100, no. 14, Article ID 141301, 2008.
[16] M. Casals and A. C. Ottewill, "Branch cut and quasinormal modes at large imaginary frequency in Schwarzschild spacetime," Physical Review D, vol. 86, no. 2, Article ID 024021, 2012.

[17] R. Banerjee and B. R. Majhi, "Quantum tunneling and trace anomaly," Physics Letters B, vol. 674, no. 3, pp. 218-222, 2009.

[18] S. W. Hawking, "The path integral approach to quantum gravity," in General Relativity: An Einstein Centenary Survey, S. W. Hawking and W. Israel, Eds., Cambridge University Press, Cambridge, UK, 1979.

[19] T. Padmanabhan, "Quasi-normal modes: a simple derivation of the level spacing of the frequencies," Classical and Quantum Gravity, vol. 21, no. 1, p. L1, 2004.

[20] A. Lopez-Ortega, "Area spectrum of the $d$-dimensional Reissner-Nordström black hole in the small charge limit ," Classical and Quantum Gravity, vol. 28, no. 3, Article ID 035009, 2011.

[21] J. Zhang and Z. Zhao, "Hawking radiation of charged particles via tunneling from the Reissner-Nordström black hole," Journal of High Energy Physics, vol. 10, p. 55, 2005.

[22] S. Hod, "Quasinormal spectrum and quantization of charged black holes," Classical and Quantum Gravity, vol. 23, no. 4, p. L23, 2006.

[23] M. K. Parikh, "A secret tunnel through the horizon," General Relativity and Gravitation, vol. 36, no. 11, pp. 2419-2422, 2004.

[24] C. Corda, "Time-Dependent Schrodinger Equation for Black Hole Evaporation: no Information Loss," http://fqxi.org/community/forum/topic/1856.

[25] C. Corda, S. H. Hendi, R. Katebi, and N. O. Schmidt, inpreparation. 

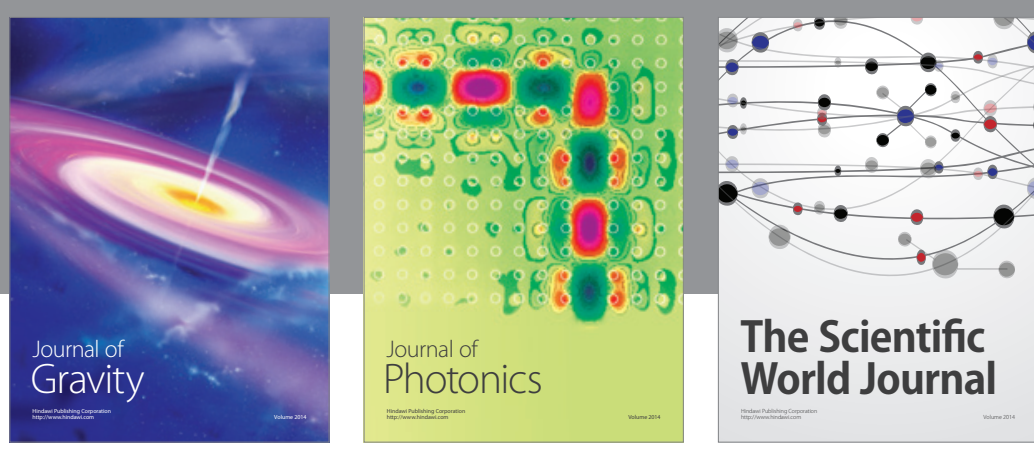

The Scientific World Journal
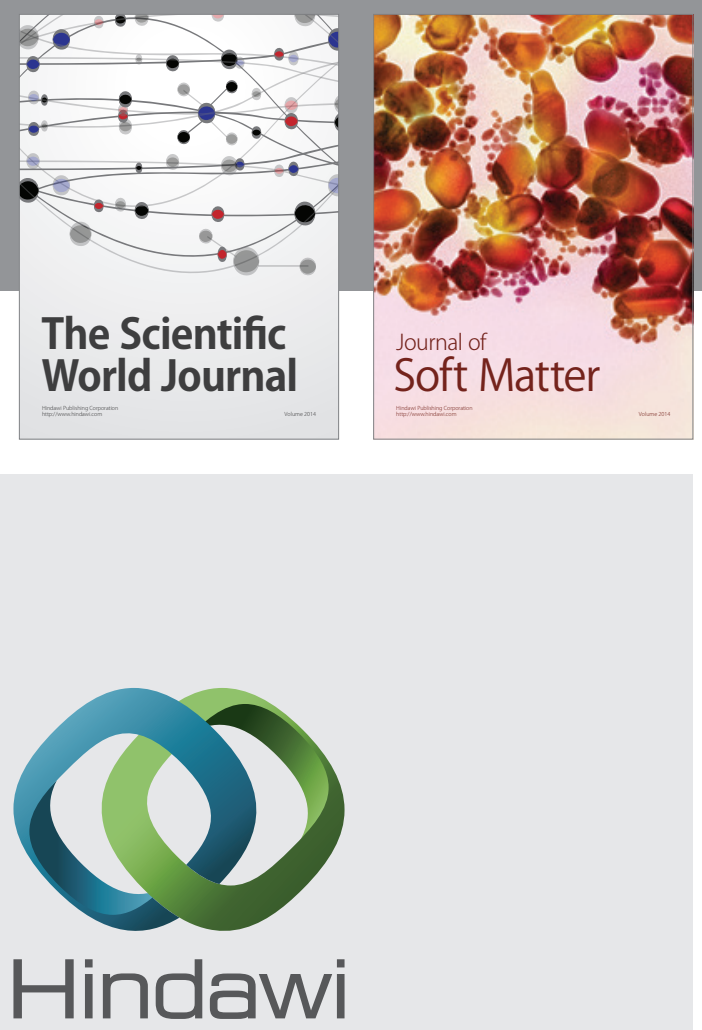

Submit your manuscripts at

http://www.hindawi.com

nternational Journal of

Statistical Mechanics
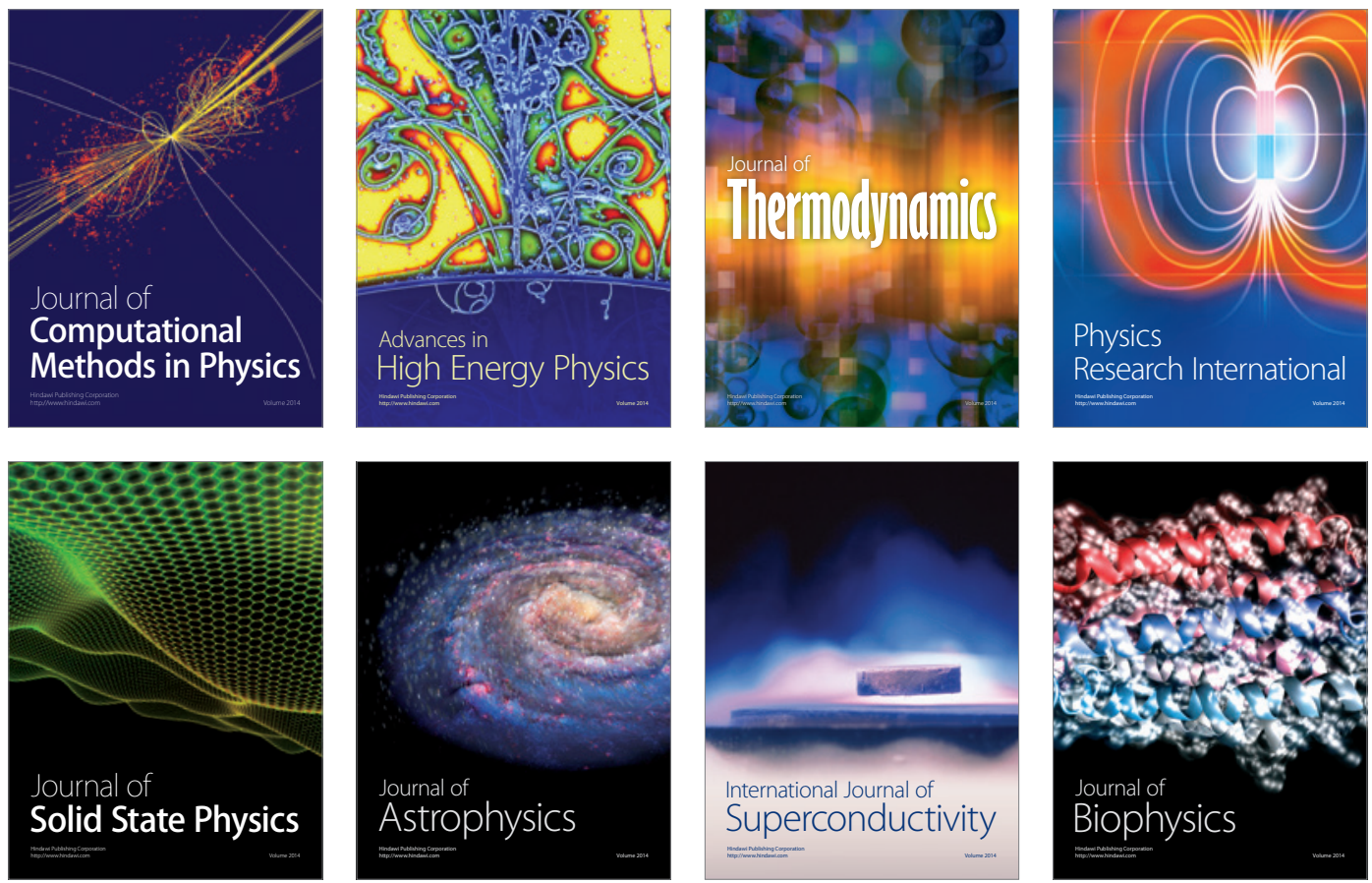
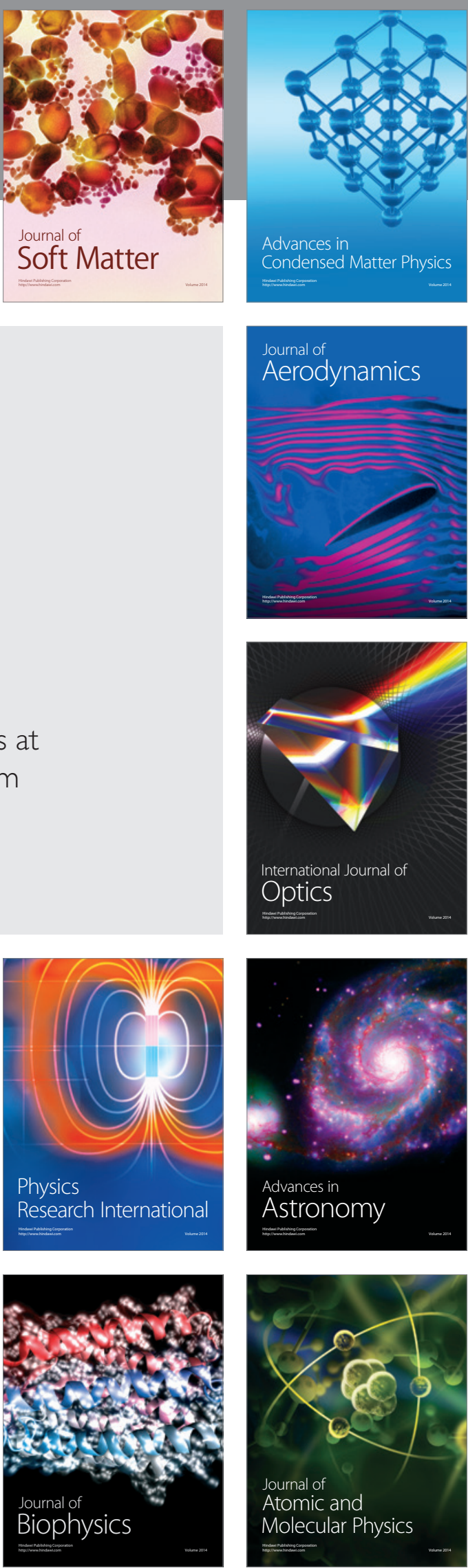